\title{
Evaluation of the appropriate size of the finite element representative volume for filled rubber composite analyses
}

\author{
Hiroshi KADOWAKI*, Gaku HASHIMOTO**, Hiroshi OKUDA**, Takeshi HIGUCHI***, \\ Hiroshi JINNAI***, Eisuke SETA* and Takanari SAGUCHI* \\ ${ }^{*}$ Central Research Division, Bridgestone Corporation \\ 3-1-1 Ogawahigashi-cho, Kodaira-shi, Tokyo 187-8531, Japan \\ E-mail: hiroshi.kadowaki@bridgestone.com \\ **Department of Human and Engineered Environmental Studies, The University of Tokyo \\ 5 Chome-1-5 Kashiwanoha, Kashiwa-shi, Chiba 277-8563, Japan \\ ***Institute of Multidisciplinary Research for Advanced Materials, Tohoku University \\ 2-1-1 Katahira, Aoba-ku, Sendai-shi, Miyagi 980-8577, Japan
}

Received 20 June 2016

\begin{abstract}
The appropriate size of the representative volume element (RVE) for filled rubber composite is investigated where the volume fraction of the filler is about $20 \%$. The micro structures of the composite are obtained by the measurement with 3-dimensional transmission electron microscope tomography (3D-TEMT) at resolution of about $1 \mathrm{~nm}$ in each direction. Numerical simulations of static elongation have been conducted by finite element method (FEM) with various sizes of RVEs where the FEM models are created by applying voxel technique to the measured data. A highly parallelized FEM program, FrontISTR, and up to 4096 cores of K computer are utilized because the largest FEM models have nearly 200-million degrees of freedom (DOFs). Two different filler dispersion states of the composite material are examined.
\end{abstract}

Key words : Nano scale composite, Filled rubber, Representative volume element, Parallel finite element method

\section{Introduction}

Filled rubber is one of the most widely used materials in engineering products such as tires, conveyor belts, seismic isolators, and etc. Enhancing the mechanical properties of this material can contribute the society through the products with improved performances. For example, increasing the elastic modulus of rubber can improve the stiffness of tire tread blocks which is important for the handling performance of a vehicle. Also, decreasing the visco-elastic loss of it can reduce rolling resistance of tires which has a significant effect to the fuel consumption of a vehicle. Filled rubber is a nano-scale composite material composed of matrix polymers, fillers and some chemical agents. Usually the matrix material is made of natural or synthetic rubber polymer, and the filler is made of carbon black or silica. The mechanical properties such as elastic or visco-elastic responses of this material are determined by interactions between such nano-scale constituents. Because filler particles in rubber compounds aggregate together to build complex and random structures, high resolution nearly $1 \mathrm{~nm}$ is required to capture the structures precisely.

Jinnai et al. $(2007,2010)$ measured the nano-scale structures of filler aggregates by 3D-TEMT. Akutagawa et al. (2008) numerically analyzed the elastic mechanical response of the captured structures by finite element method (FEM) where the resolution was about $4 \mathrm{~nm}$ and the domain size was approximately in the range of 120 to $260 \mathrm{~nm}$. This domain size and resolution were appropriate when the volume fraction of the filler particles was less than $15 \%$. However, considering the rubber compound utilized in the real world products where the fraction is more than $20 \%$, authors found that the resolution is not fine enough to capture the complex 3D structure of filler aggregations, and the domain size is not large enough to obtain the representative response of the composite ( Kadowaki et al. 2014a, 2014b). Because the distributions of filler aggregations are so random, the stress-strain response of FEM results fluctuates a lot 
with the choice of the area for analysis. Also, FEM analyses with coarse resolution yields stiff responses because they cannot capture the narrow gaps between filler aggregates. Figure 1 shows one section of filled rubber with about $20 \%$ filler volume fraction measured at resolution of $1 \mathrm{~nm}$ by $\mathrm{H}$. Jinnai, who is one of the authors. One can find that (1) the resolution should be nearly $1 \mathrm{~nm}$ because the distances between filler aggregates are several nanometers, and (2) the domain size should be as large as several hundred nanometers because the distributions of filler particles are non-uniformly located. Other researchers also take the analysis domain size which is larger than that of Akutagawa et al. (2008). For example, Kishimoto, et al. (2013) chose the domain size 459 x 712 x $489 \mathrm{~nm}$ for their FEM analysis of filled rubber. Kishimoto (2015) estimates the representative volume size as 350 x 350 x $350 \mathrm{~nm}$ for their coarse-grained molecular dynamics analysis considering the size of secondary aggregate of silica filler. Koishi (2015) and Koishi et al. (2015) chose the domain size as 1000 x 1000 x 1000nm for their analyses. However there are scarce works which investigate the size of RVE systematically and quantitatively.

In this paper, our focus is to investigate the appropriate size of RVE for filled rubber with $20 \%$ filler volume fraction. In order to evaluate the appropriate size of RVE, we need a reference solution with sufficiently large domain size. Thus we conducted FEM analyses with various sizes of domain up to $770 \times 820 \times 117$ nm, which we call "the reference domain size". The nominal stresses of RVEs are compared with measurements. Because the FEM model size becomes nearly 200 million degree of freedoms, the computations are conducted with FrontISTR which is a highly parallelized FEM program, and with up to 4096 cores of K-computer. Basic idea of parallel FEM employed in FrontISTR is found in Hashimoto and Okuda (2014), Kushida and Okuda (2014), Okuda and Yagawa (2005), Okuda et al.(2003), and Nakajima and Okuda(1999). Program sources and related information can be found in the web site of FrontISTR Forum (Okuda, 2015).

In section 2, we describe the filler structure data for filled rubber measured by 3D-TEMT (Jinnai et al., 2007, NEDO, 2012) and how the FEM analysis models are created. In section 3, the element formulation is explained which can avoid the volumetric locking behavior due to the incompressibility of the matrix material. In section 4, large scale FEM analyses for the reference domain size are shown with the performance on K-computer. In section 5, elastic responses of RVEs with various sizes are compared with measurement data to investigate the appropriate size of RVE. In section 6, we discuss the reason of appropriate size of RVE and the gap between FEM analyses and the measurements. In section 7 , summary and concluding remarks are given.

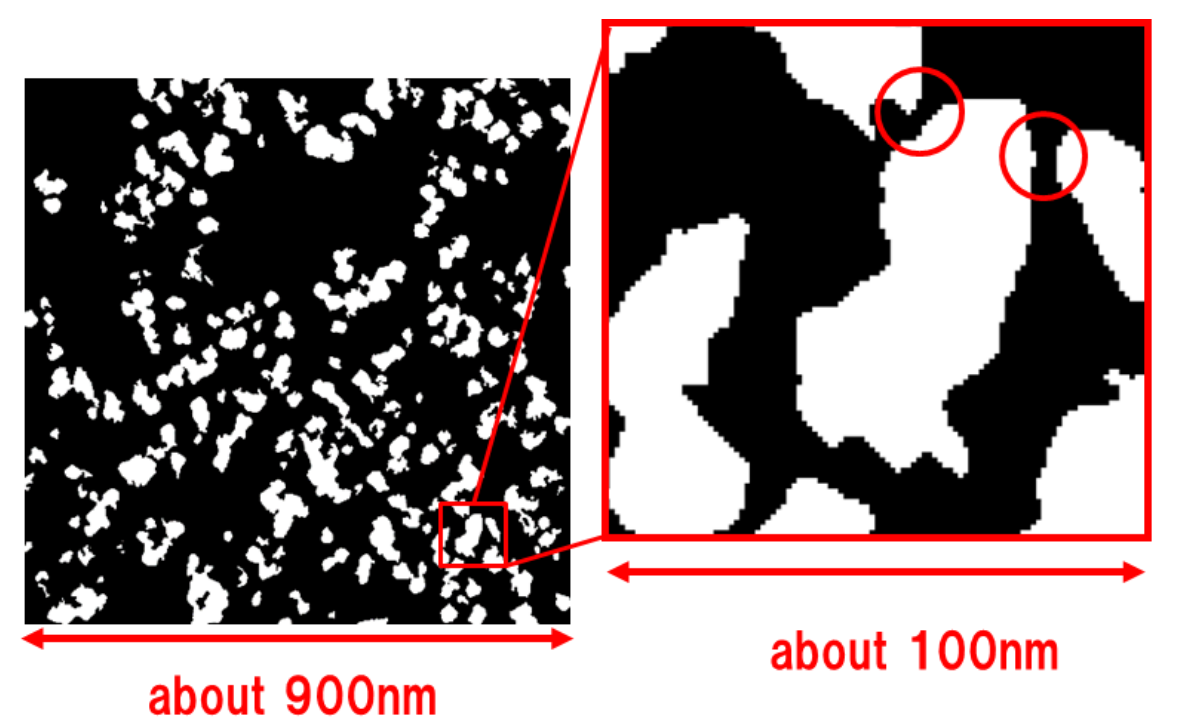

Figure 1 One section of a filled rubber composite measured by 3D-TEMT is shown. Filler volume fraction is about 20\%. White pixels show the filler part and black pixels show the matrix polymer part.

\section{FEM models of filled rubber composite}

Two different sets of filler structures, Filler Structure A and B, are prepared. Filler Structure A shows conventional dispersion and Filler Structure B shows more uniform dispersion of filler particles (Fig. 2). Filler volume fractions of 
these structures are $20.5 \%$ and $23.0 \%$ respectively. Their 3D structures were measured by 3D-TEMT. The size of the domain is $770 \times 820 \times 117 \mathrm{~nm}$, and the resolutions are $850 \times 850 \times 91$ pixels. FEM models are created by so-called voxel meshing technique. In this case one pixel of the images is converted to one voxel, which is an 8-noded hexahedral finite element (Fig. 3). Therefore the FE model is composed of $850 \times 850$ x 91 elements and $851 \times 851 \times 92$ nodes. Because each node has three DOFs, the total number of DOFs becomes nearly 200 million.
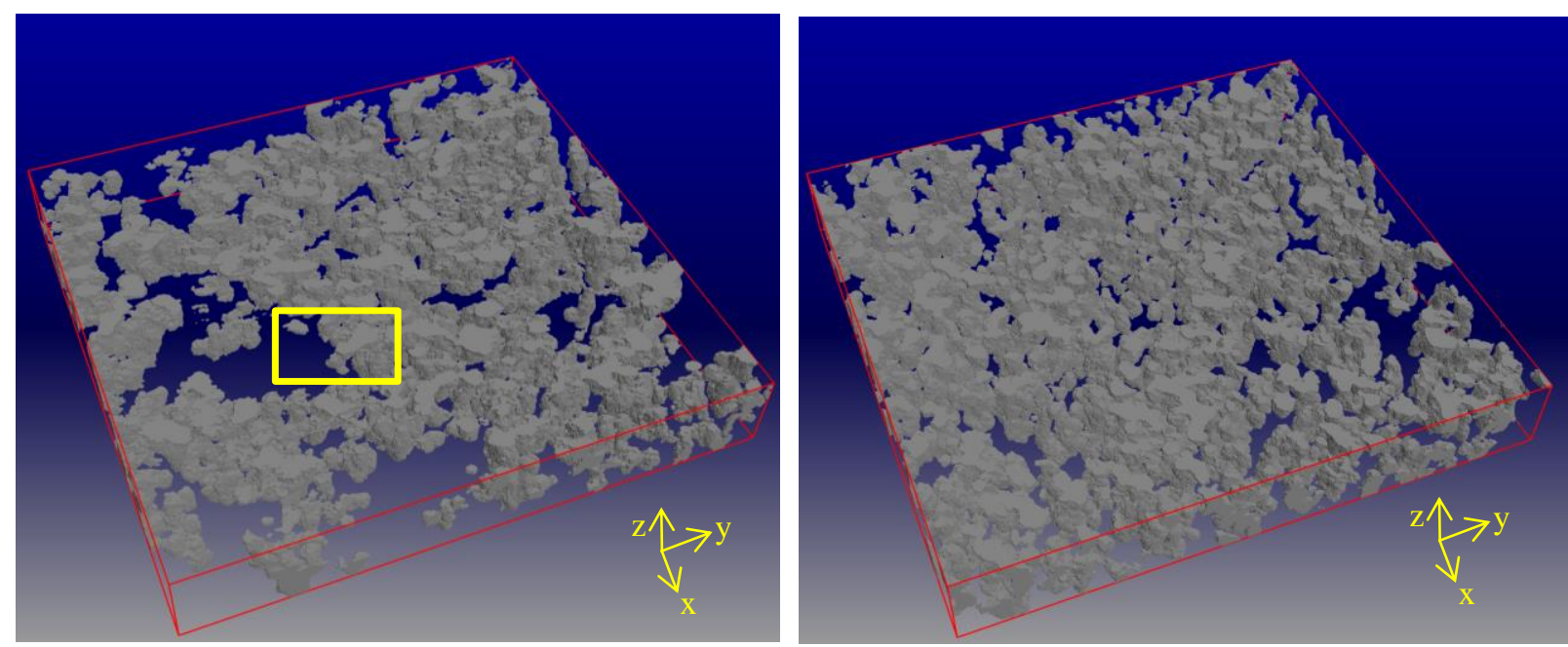

Figure 2 The FEM models of the two sets of filler structures with different dispersion state. Left figure shows the conventional filler dispersion (Filler Structure A), and right figure shows more uniform dispersion (Filler Structure B). The volume fractions of filler for these structures are $20.5 \%$ and $23.0 \%$ respectively. The zoomed image of the area marked by the yellow rectangle is shown in Fig.3.

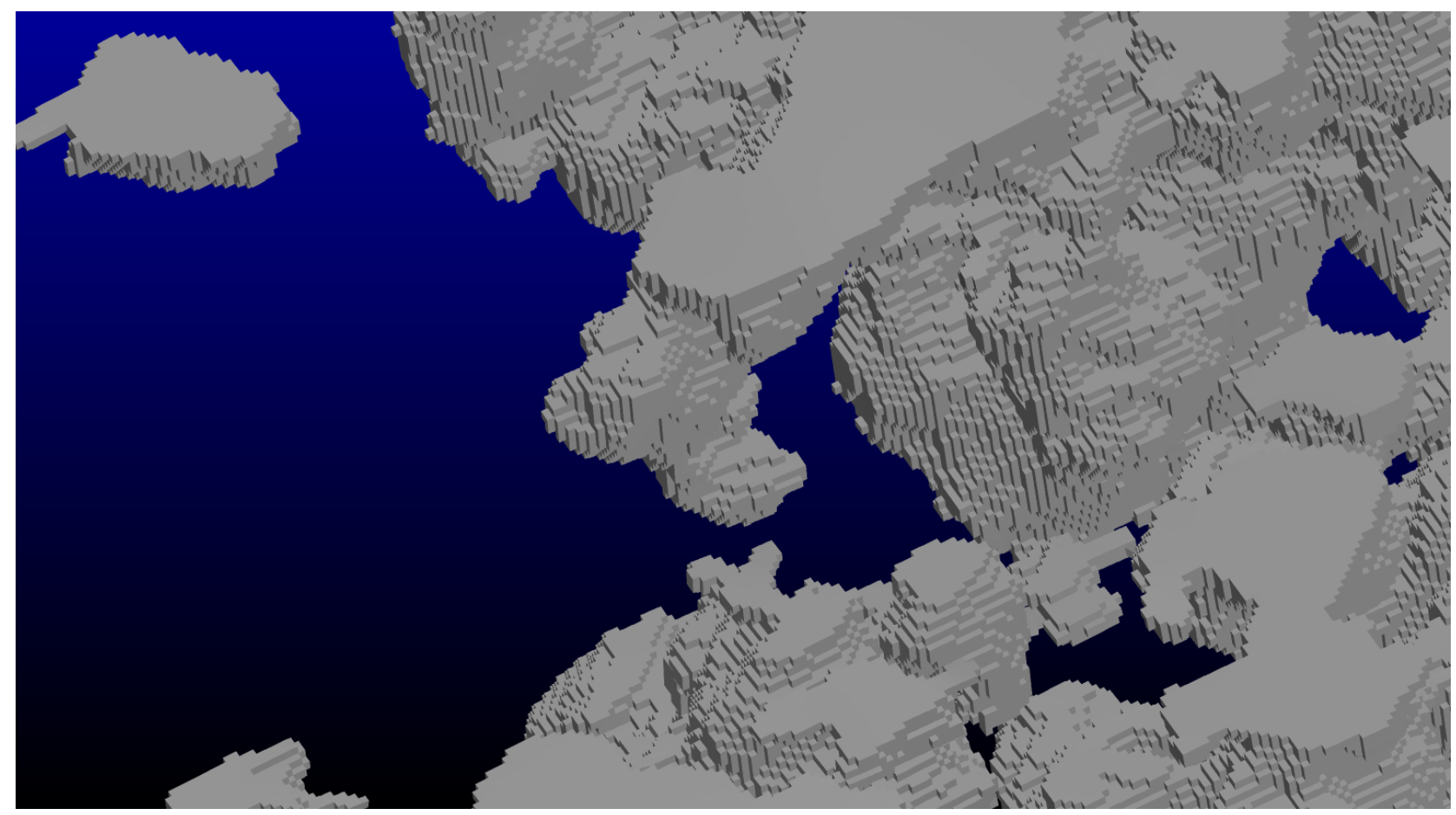

Figure 3 Zoomed image of the yellow rectangle area of the left figure of Fig. 2 is shown to explain the discretization with the voxel technique.

\section{Element formulation for nearly incompressible material}

Rubber is known to be a nearly incompressible material. When we analyze such material by FEM, we need to use a 
special element formulation to avoid volumetric locking. The boundary condition applied to the RVE in this research is uniaxial stress condition which does not cause volumetric locking in a macroscopic view. However, there are highly constrained areas inside the composite where volumetric locking may occur, especially in narrow matrix polymer regions between fillers. Thus, u/p mixed formulation element (Sussman and Bathe, 1987) is implemented for FrontISTR and compared to B-bar method (Hughes, 1980) which is the default element in this FEM program. Because authors followed the formulation of Sussman and Bathe (1987), only the choices of strain energy functions are briefly explained in this section.

Let $I_{1}, I_{2}$, and $I_{3}$ be the first, second and the third invariants of the right Cauchy-Green deformation tensor defined by the deformation gradient, $\mathbf{F}$, as:

$$
\begin{aligned}
& \mathbf{C}=\mathbf{F}^{T} \mathbf{F} \\
& I_{1}=\operatorname{tr}(\mathbf{C}) \\
& I_{2}=\frac{1}{2}\left\{(\operatorname{tr}(\mathbf{C}))^{2}-\operatorname{tr}\left(\mathbf{C}^{2}\right)\right\} \\
& I_{3}=\operatorname{det}(\mathbf{C})
\end{aligned}
$$

In order to separate the isochoric deformation and volumetric deformation, this formulation utilizes the reduced invariants $\tilde{I}_{1}, \tilde{I}_{2}$, and $J$ defined as follows:

$$
\begin{aligned}
& \tilde{I}_{1}=I_{1} I_{3}^{-1 / 3} \\
& \tilde{I}_{2}=I_{2} I_{3}^{-2 / 3} \\
& J=I_{3}^{1 / 2}
\end{aligned}
$$

The strain energy density functions for the isochoric deformation $W\left(\tilde{I}_{1}, \tilde{I}_{2}\right)$ and volumetric deformation $g(J)$ are defined as follows:

$$
\begin{aligned}
W\left(\tilde{I}_{1}, \tilde{I}_{2}\right) & =C_{10}\left(\tilde{I}_{1}-3\right)+C_{01}\left(\tilde{I}_{2}-3\right) \\
g(J) & =\frac{1}{D}(J-1)^{2}
\end{aligned}
$$

where $C_{10}, C_{01}$ and $\mathrm{D}$ are material parameters. They are shown in Table 1 . Note that the material parameter $\mathrm{D}$ is computed from $C_{10}, C_{01}$ and Poisson's ratio $v$ as follows:

$$
\begin{aligned}
& G_{0}=2\left(C_{10}+C_{01}\right) \\
& K_{0}=\frac{2 G_{0}(1+v)}{3(1-2 v)} \\
& D=\frac{2}{K_{0}}
\end{aligned}
$$

where $G_{0}$ and $K_{0}$ are shear modulus and bulk modulus at the non-deformed state respectively.

The material response for this strain energy density function are computed at $2 \times 2 \times 2$ integration points for a 8-node hexahedral element assuming that hydrostatic pressure is constant over the element. The performance of this element is tested with a beam bending problem shown in Fig. 4 with various Poisson's ratios shown in Table 1. The parameters $C_{10}$ and $C_{01}$ are obtained from uniaxial tension experiment of unfilled SBR polymer. The domain is discretized by $8 \times 2$ elements. Figure 5 shows the results of both B-bar formulation and u/p mixed formulation. The displacements at the loading point, $\mathrm{P}$, are normalized by the solution which is obtained with a finely discretized model ( $400 \times 100$ elements) where both formulations yield almost identical result without any clear locking behaviors. One can find that $\mathrm{u} / \mathrm{p}$ mixed formulation does not show any locking behavior in these analyses whereas B-bar formulation exhibit locking behavior when Poisson's ratio is larger than 0.499 . 
Table 1 Material Parameters for the Mooney-Rivlin model.

\begin{tabular}{|l|l|}
\hline$C_{10}$ & $0.2826 \times 10^{6}[\mathrm{~Pa}]$ \\
\hline$C_{01}$ & $0.01448 \times 10^{6}[\mathrm{~Pa}]$ \\
\hline$v$ & $0.4,0.45,0.49,0.499,0.4999,0.49999$ \\
\hline
\end{tabular}

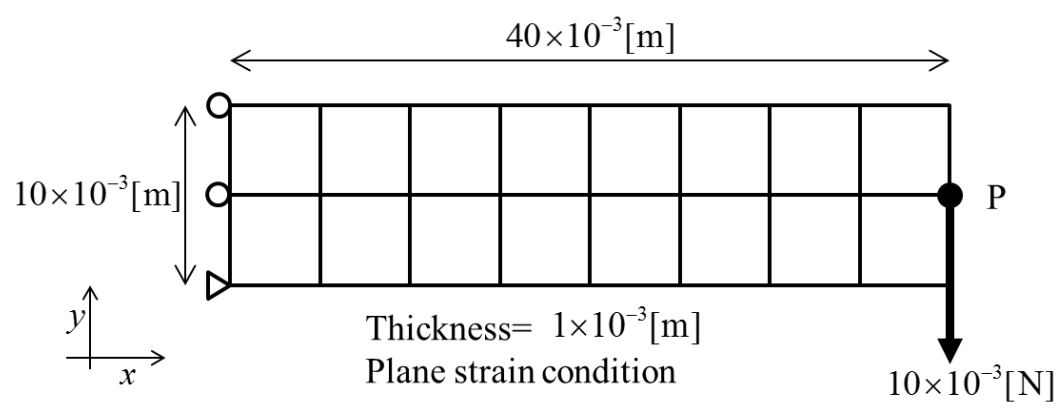

Figure 4 A schematic view of beam bending problem (JSCE, 2008) is shown. The domain is discretized by $8 \times 2$ elements. A concentrated load is applied at point $\mathrm{P}$ in the $\mathrm{y}$-direction. The displacements at point $\mathrm{P}$ in the $\mathrm{y}$-direction are compared.

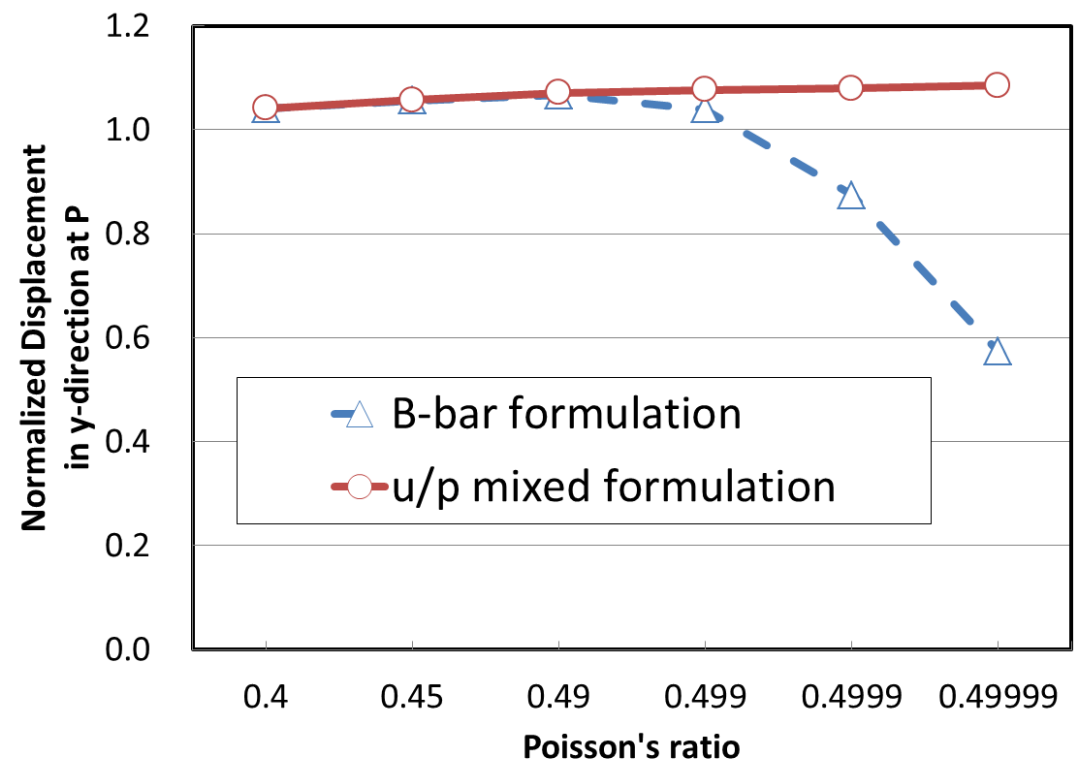

Figure 5 Results of beam bending analyses shown in Fig. 4. B-bar formulation and u/p mixed formulation are compared with various Poisson's ratios. The displacements are normalized by the results obtained with a finely discretized model.

\section{FEM computation for the entire domain of the measured data as the reference size RVEs}

The FEM models of the reference domain size shown in section 2 are analyzed by the FEM program FrontISTR. Matrix polymers are modeled by implementing the elements with $\mathrm{u} / \mathrm{p}$ mixed formulation explained in section 3 . The material parameters for filler and matrix are shown in Table 2. The entire domain is stretched in the y-direction shown in Fig. 2. Figure 6 shows the distribution of the first principal strain when 5\% nominal strain is applied. Because the dispersion of filler in Filler Structure A is relatively non-uniform, one can find small strain level in the region where filler particles are sparsely distributed, whereas one can find high strain level between fillers where filler particles are densely distributed. On the other hand, strain is more uniformly distributed for filler structure B because of the uniform 
dispersion of filler particles.

Because the total number of DOFs is nearly 200 Million, K computer developed by RIKEN is utilized. Table 3 shows the computational performance measured on K computer with the domain size of 850 x 850 x 91 elements. More than 2TFLOPS and $60 \%$ parallel efficiency have been attained. As the number of cores is increased, the ratio to the peak performance decreases. The reason is that, as the number of cores increases, the amount of calculation per core decreases and communication between cores increases.

Table 2 Material constants for filler and matrix polymer

\begin{tabular}{|l|l|l|}
\hline $\begin{array}{l}\text { Filler } \\
\text { (St.Venant-Kirchhoff } \\
\text { material) }\end{array}$ & Young's Modulus & $0.45 \times 10^{9}[\mathrm{~Pa}]$ \\
\cline { 2 - 3 } $\begin{array}{l}\text { Matrix polymer } \\
\text { (Mooney-Rivlin } \\
\text { hyperelastic material) }\end{array}$ & Poisson's Ratio & 0.2 \\
\cline { 2 - 3 } & $\mathrm{C}_{10}$ & $0.283 \times 10^{6}[\mathrm{~Pa}]$ \\
\cline { 2 - 3 } & $\mathrm{C}_{01}$ & $0.0145 \times 10^{6}[\mathrm{~Pa}]$ \\
\cline { 2 - 3 } & $\mathrm{D}$ & $67.3 \times 10^{-9}[1 / \mathrm{Pa}]$ \\
\hline
\end{tabular}
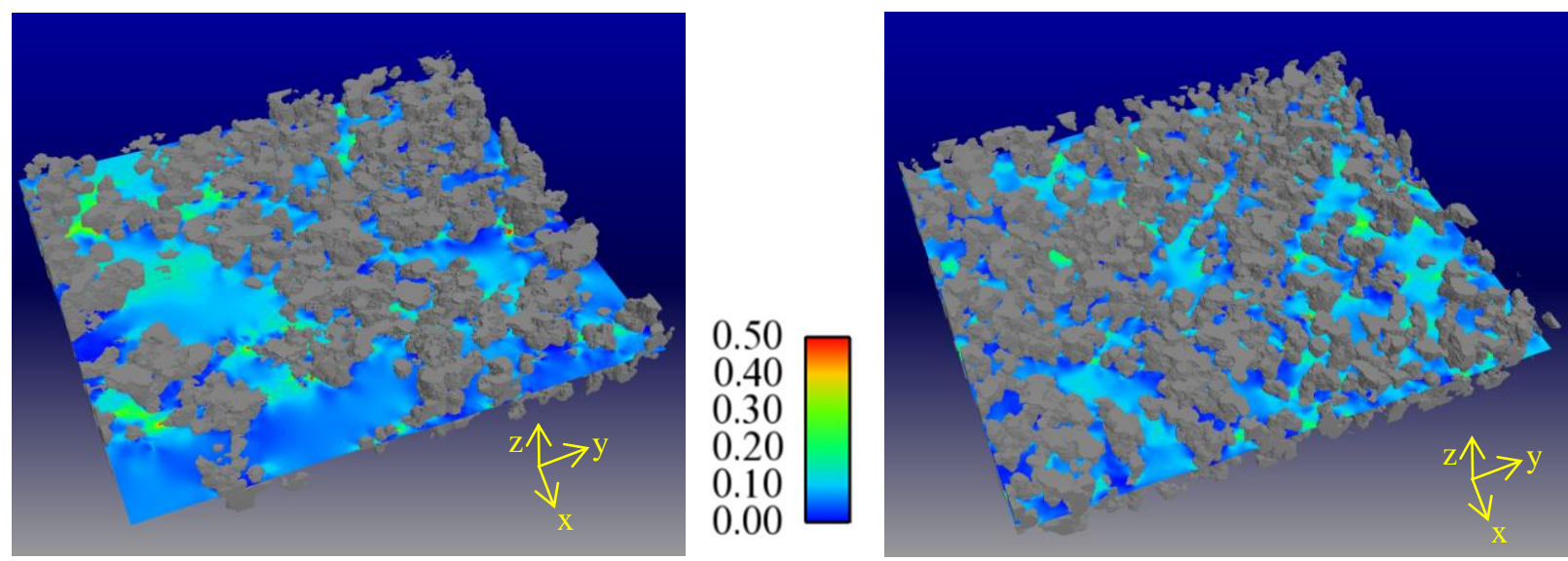

Figure 6 Distribution of the first principal value of strain (left: Filler Structure A, right: Filler Structure B).

Table 3 Computational Performances of FrontISTR (flat MPI) with u/p mixed formulation elements on K-computer with the model size of nearly 200 million DOFs.

\begin{tabular}{|c|c|c|c|}
\hline Number of Cores & TFLOPS & $\begin{array}{l}\text { Ratio to } \\
\text { the Peak Performance }\end{array}$ & $\begin{array}{l}\text { Parallel } \\
\text { Efficiency (\%) }\end{array}$ \\
\hline 1,024 & 0.89 & 5.4 & --- \\
\hline 2,048 & 1.51 & 4.6 & 68.7 \\
\hline 4,096 & 2.50 & 3.8 & 64.8 \\
\hline
\end{tabular}

\section{Investigation of the RVE size}

In order to investigate the appropriate RVE size for filled rubber of $20 \%$ filler volume fraction, FEM analyses have been conducted with various sizes of RVEs. The size of FEM models explained in section 4 is set as the reference domain size $(770 \times 820 \times 117 \mathrm{~nm})$. Then, keeping the resolution of the analysis, this FEM model was divided into $2 \times 2$ sub-models in the $\mathrm{x}$ - and y-directions allowing some overlaps between each sub-model which is shown in Fig. 7. In this case, one sub-model is the size of $544 \times 579 \times 117 \mathrm{~nm}$ and it is considered as the RVE. Note that the length in the z-direction of the domain was kept constant in order to avoid getting a model size smaller than 90nm in one direction. In the same manner, the FEM model of the reference domain size was divided into $3 \times 3,5 \times 5$, and $10 \times 10$ sub-models shown in Table 4. In this table, the sizes of RVE are defined according to the approximate sub-model size such as $800 \mathrm{~nm}, 560 \mathrm{~nm}$, and so on. 

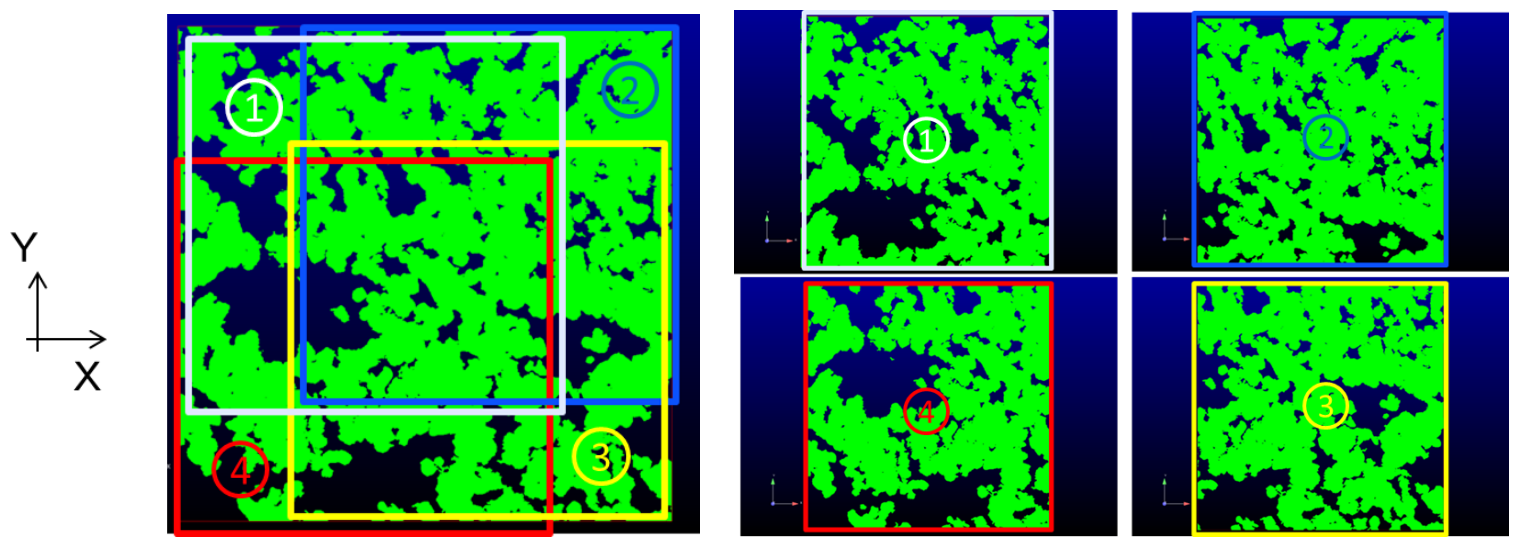

Figure 7 A schematic view showing how the reference size RVE was divided into 2 x 2 sub-models with overlaps.

Table 4 the sizes, numbers of elements, and the number of sub-models

\begin{tabular}{|l|l|c|c|c|}
\hline $\begin{array}{l}\text { RVE } \\
\text { size }\end{array}$ & Division & Size of a sub-model(nm) & $\begin{array}{c}\text { Number of elements in each } \\
\text { direction }\end{array}$ & Number of sub-models \\
\hline $800 \mathrm{~nm}$ & $1 \times 1$ & $770 \times 820 \times 117$ & $850 \times 850 \times 91$ & 1 \\
\hline $560 \mathrm{~nm}$ & $2 \times 2$ & $544 \times 579 \times 117$ & $600 \times 600 \times 91$ & 4 \\
\hline $370 \mathrm{~nm}$ & $3 \times 3$ & $362 \times 386 \times 117$ & $400 \times 400 \times 91$ & 9 \\
\hline $190 \mathrm{~nm}$ & $5 \times 5$ & $181 \times 193 \times 117$ & $200 \times 200 \times 91$ & 25 \\
\hline $90 \mathrm{~nm}$ & $10 \times 10$ & $91 \times 96 \times 117$ & $100 \times 100 \times 91$ & 100 \\
\hline
\end{tabular}

Figure 8 shows the elastic response of the composite FEM models with various RVE sizes. The plots for 90, 190, 370 , and 560nm are the average of the partially overlapped RVEs of the same size. Firstly, one can notice that larger RVE can predict more accurate response of composites. Secondly, one can notice that the responses of Filler Structure A, conventional dispersion, tend to divert from that of the reference domain when the RVE size is smaller than 370nm. On the other hand, the responses of Filler Structure B tend to divert less than those of Filler Structure A, because the dispersion is more uniform in this structure. Although there is a gap between measurement and FEM prediction in the absolute value of the stress, the relative value shows good coincidence when the RVE size is above 370nm (Fig. 9).
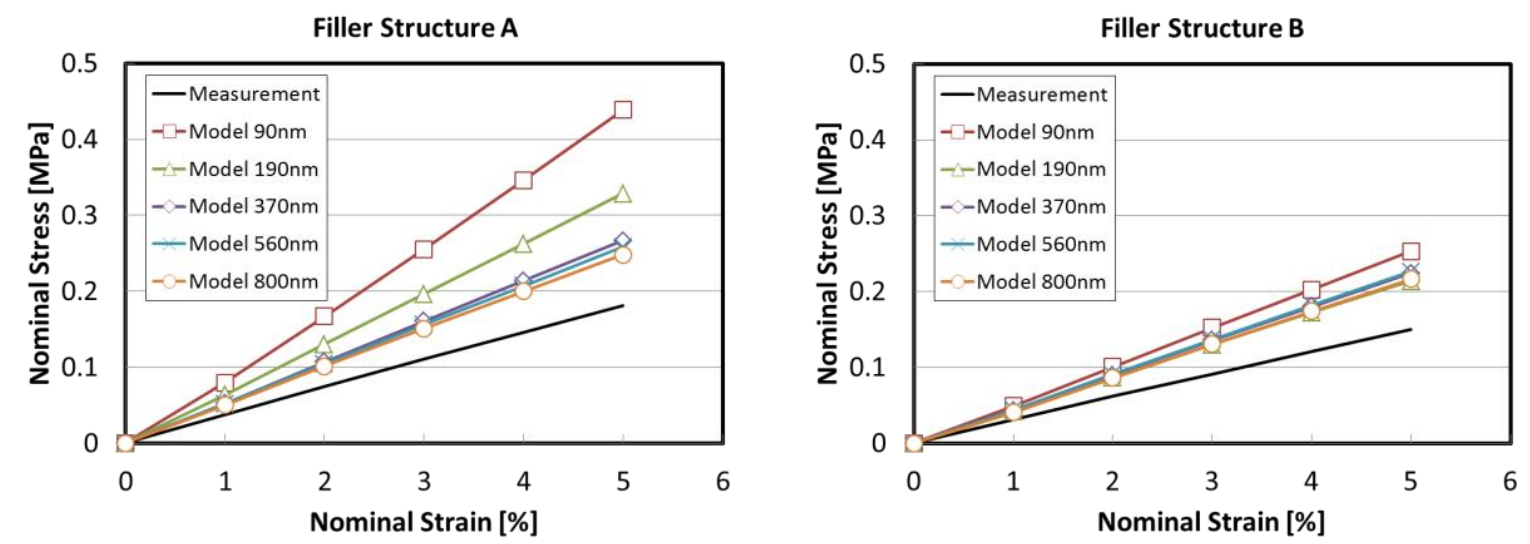

Figure 8 Nominal stress vs nominal strain of the composite with various model sizes. Data for model 90nm, 190nm, $370 \mathrm{~nm}$, and 560nm are the average of sub-models. Left: Filler Structure A, right: Filler Structure B. 


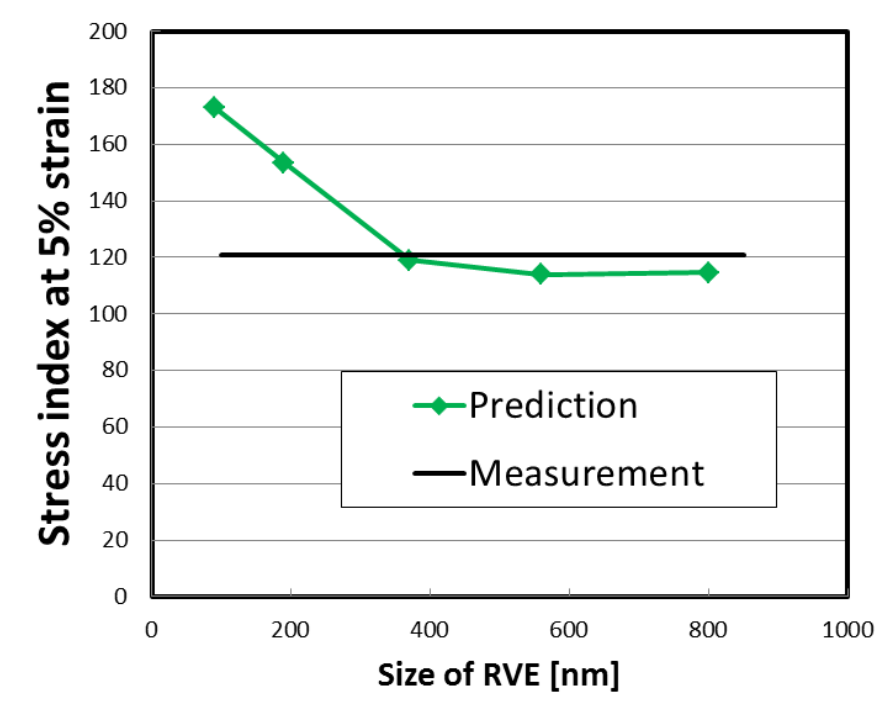

Figure 9 Comparison in relative value of stress. The index is calculated by the stress of Filler Structure A divided by that of Filler Structure B at $5 \%$ strain.

\section{Discussion}

We note that the previous section investigates the appropriate RVE size for filled rubber of about $20 \%$ filler volume fraction. When the RVE size is smaller than 370nm, FEM yields higher stress than that of reference domain size for the Filler Structure A. For example the stress is $200 \%$ higher when RVE size of $90 \mathrm{~nm}$ is taken. One reason for this phenomenon is that the RVE size is not large enough compared to the size of filler aggregates and both sides of RVEs are connected by stiff filler material. Figure 10 shows the distribution of the stress of each RVEs of $90 \mathrm{~nm}$ at $5 \%$ strain for Filler Structure A and B. For both Filler Structures, a majority of data distributes within a small deviation from a peak value. The peak values locate between 0.2 and $0.25 \mathrm{MPa}$, which coincides with the results of the reference domain size. The small deviation from the peak values can be explained by the fluctuation of filler volume fraction among RVEs. However, for Filler Structure A, a notable portion of the data shows much higher stress. For example about $10 \%$ of the RVEs yield more than 1MPa. It cannot be explained by the filler volume fraction. Because of these high stress data, the average stress of Filler Structure A largely deviates from that of reference domain size. Figure 11 shows two of the 90nm-sized RVEs for Filler Structure A which yield the highest and the second highest stress values. One can find that the both sides of the RVEs are connected by stiff filler material in the loading direction and it makes the RVEs very stiff. As the RVE size becomes smaller and as the filler aggregate becomes larger, these stiff RVEs are found more frequently and it makes the average stress higher.

Even though the RVE size is larger than $370 \mathrm{~nm}$, the absolute values of stress obtained by FEM analyses are about $40 \%$ higher than the measurement. One possible reason for this discrepancy is the simplified FEM modelling of the filler-matrix interfaces. In the FEM model of this study, filler and matrix are perfectly bonded by sharing the same node. However it is known that there exist slips and separations on the interfaces in molecular scale. In order to reduce the discrepancy between FEM and measurement, one possible remedy is to develop a more precise interface model so that slips and separations are incorporated in the analysis. 


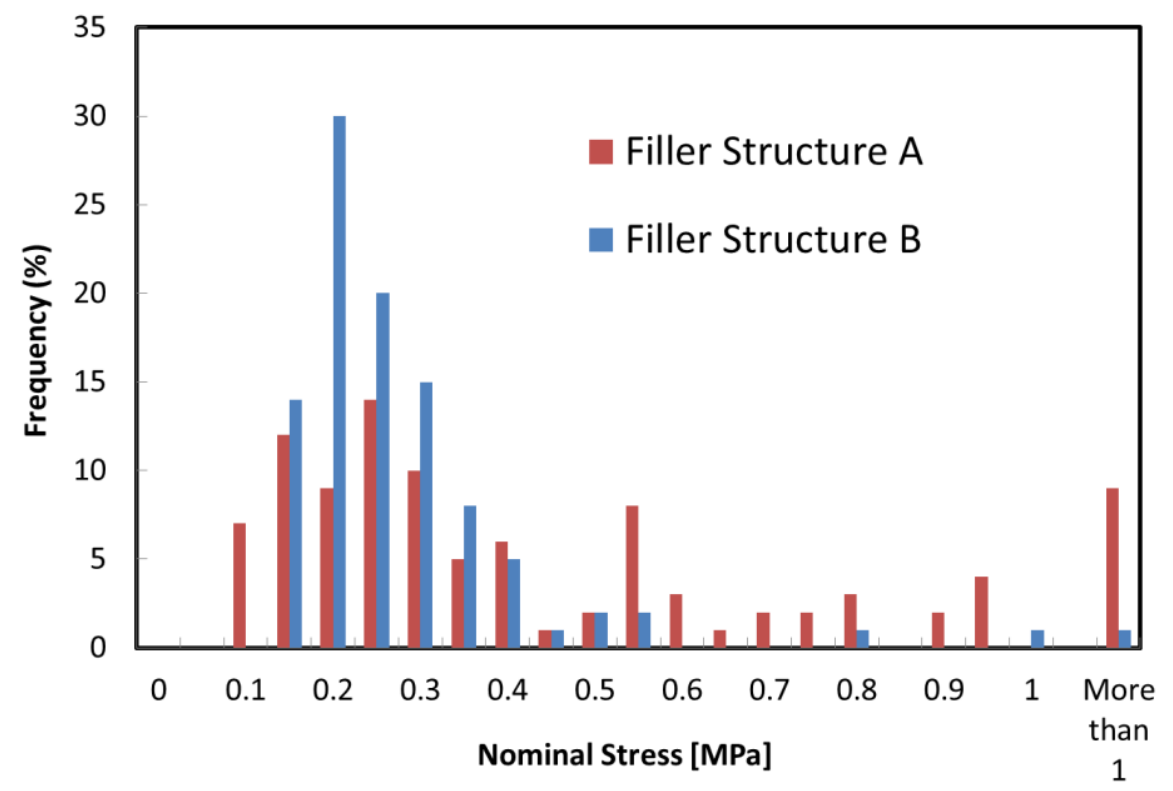

Figure 10 Histogram of nominal stress values at 5\% strain for RVEs sized 90nm

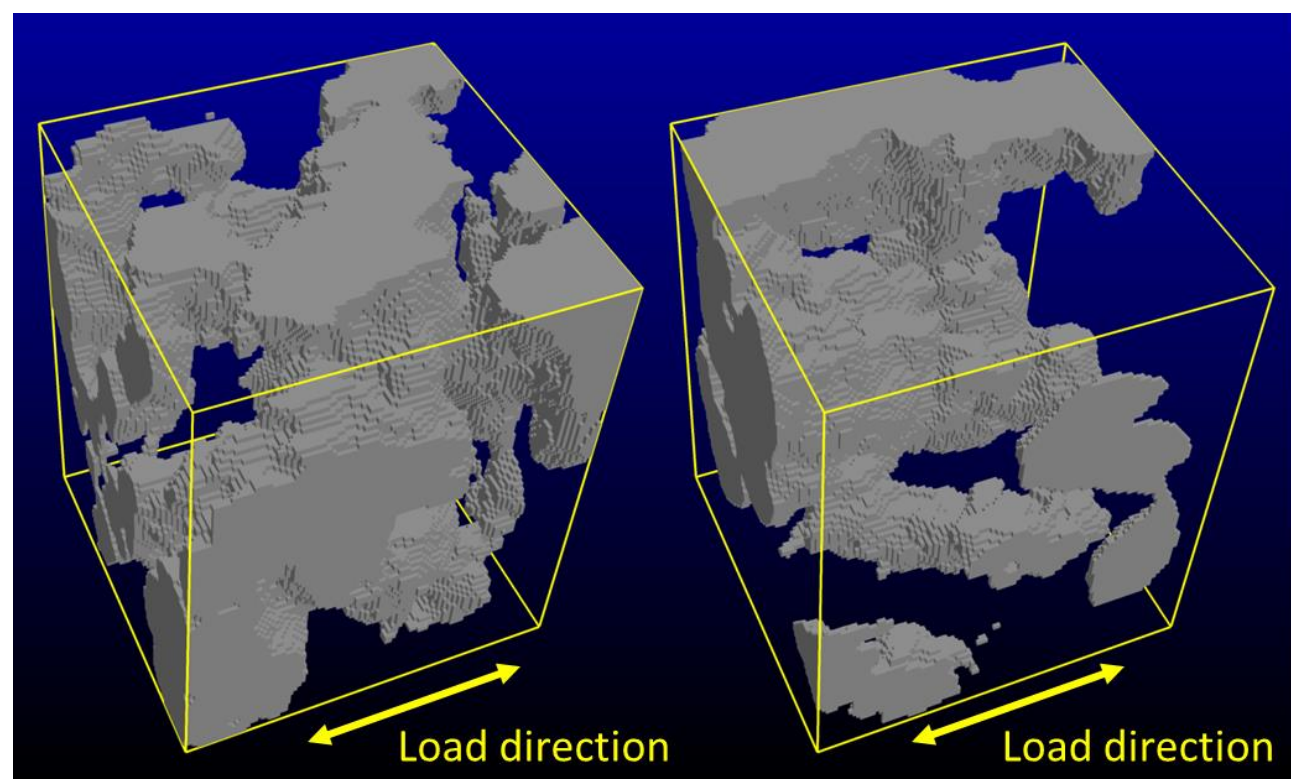

Figure 11 Filler structure of 90nm size RVEs, which yields the highest and the 2nd highest stress for Filler Structure A. The nominal stress values are 1.7MPa and 1.6MPa for the left and the right model, respectively.

\section{Summary and Conclusions}

The appropriate size of RVE is investigated for filled rubber composite with $20 \%$ filler volume fraction. FEM analyses are conducted over various sizes of RVE sizes up to 770 × 820 × $117 \mathrm{~nm}$ with the resolution of about $1 \mathrm{~nm}$ in each direction. It is found that about $370 \mathrm{~nm}$ is required to obtain the representative elastic response. When the size of RVE is smaller than that, the average stress of RVEs may become higher than that of larger RVEs. It is because a filler aggregate connects both sides of the RVEs in the loading direction in a considerable portion of RVEs. There is a good coincidence in the relative value of stress between two different dispersion states. However, there is a discrepancy between predictions and measurements in the absolute value of the stress even though one takes a sufficiently large size of RVE. One reason for this discrepancy is that filler material and matrix material are perfectly bonded in the FEM model of this study. Further research with more precise interface models is needed to explain this.

The conclusions in the previous paragraph can only be made with reference solutions obtained by sufficiently large 
analysis domain. The model size for these reference solutions becomes nearly 200million DOFs and it can only be solved by high performance computers with several thousands of CPU cores. We conducted those large scale analyses with 4096 cores of the K-computer. More than 2 TFlops performance and 60\% parallel efficiency are attained.

\section{Acknowledgements}

This research used computational resources of the $\mathrm{K}$ computer provided by the RIKEN Advanced Institute for Computational Science through the HPCI System Research project (Project ID:hp120119). This research used the measured data of filler microstructures which are obtained by New Energy and Industrial Technology Development Organization (NEDO) project (Project ID: 09002153-0).

\section{References}

Akutagawa K., Yamaguchi K., Yamamoto A., Heguri H., Jinnai H., and Shinbori Y., Mesoscopic Mechanical Analysis of Filled Elastomer with 3D-Finite Element Analysis and Transmission Electron Microtomography, Rubber Chemistry and Technology, Vol. 81, No. 2 (2008), pp. 182-189.

Hashimoto G., and Okuda H., Large-scale one-way coupled analysis with open-source parallel FEM software FrontISTR , NAFEMS European Conference on Multiphysics Simulation 2014 (2014), pp. 31-33.

Hughes T. J. R., Generalization of selective integration procedures to anisotropic and nonlinear media, International Journal for Numerical Methods in Engineering, Vol. 15, Issue 9 (1980), pp 1413-1418.

Jinnai H., Shinbori Y., Kitaoka T., Akutagawa K., Mashita N., and Toshio Nishi, Three-Dimensional Structure of a Nanocomposite Material Consisting of Two Kinds of Nanofillers and Rubbery Matrix Studied by Transmission Electron Microtomography, Macromolecules, Vol. 40 (2007), pp. 6758-6764.

Jinnai H., Spontak R. J., and Nishi T., Transmission Electron Microtomography and Polymer Nanostructures, Macromolecules, Vol. 43 (2010), pp. 1675-1688.

Japan Society of Civil Engineers (JSCE) ed., Common Sense in Computational Mechanics (2008), p.64, Maruzen (in Japanese).

Kadowaki H., Hashimoto G., Okuda H., Seta E., and Heguri H., A Large Scale Parallel FEM Analysis for Filled Rubber Material Considering its Micro-scale Structures, Proceedings of the Conference on Computational Engineering and Science, Vol. 19 (2014a) (in Japanese).

Kadowaki H., HPCI user report, project ID: hp120119 (2014b) (in Japanese).

Kishimoto H., Naito M., Ueno S., Shinohara Y., Amemiya Y., Application of Quantum Beam and Simulation on Analysis of Polymer Composite, Proceedings of JSME 26th Computational Mechanics Division Conference (2013) (in Japanese).

Kishimoto H., HPCI user report, project ID: hp120032 (2015) (in Japanese).

Koishi M., Simulation to produce new ideas, JSME Mechanical Engineering Congress (2015) (in Japanese).

Koishi M., Kowatari N., Willot F., and Jeulin D., Large scale viscoelastic simulation of filled rubber by FFT-based schemes, Proceedings of JSME 28th Computational Mechanics Division Conference (2015) (in Japanese).

Kushida N. and Okuda H., Optimization of the Parallel Finite Element Method for the Earth Simulator, Journal of Computational Science and Technology, Vol.2, No.1 (2008), pp. 81-91.

Nakajima K. and Okuda H., Parallel Iterative Solvers with Localized ILU Preconditioning for Unstructured Grids, Iterative Methods in Scientific Computation IV, IMACS Series in Computational and Applied Mathematics, Vol. 5 (1999), pp. 85-98.

New Energy and Industrial Technology Development Organization (NEDO), Elastomer with three-dimensional nano-scale hierarchical structure optimized for super fuel efficient tires, NEDO Report, Number: 20120000000875 (2012) (in Japanese).

Okuda H., Nakajima K., Iizuka M., Chen L., and Nakamura H., Parallel Finite Element Analysis Platform for the Earth Simulator:GeoFEM, Proc. Computational Science-ICCS2003, LNCS2659, PartIII (2003), pp. 773-780.

Okuda H. and Yagawa G., Large-Scale Parallel Finite Element Analysis for Solid Earth Problems by GeoFEM, Surveys on Mathematics for Industry, Vol.11, No.1-4 (2005), pp. 159-196.

Okuda H., FrontISTR Forum (2015) (online), available from <http://www.multi.k.u-tokyo.ac.jp/FrontISTR/index_en.php>. 
Kadowaki, Hashimoto, Okuda, Higuchi, Jinnai, Seta and Saguchi, Mechanical Engineering Journal, Vol.3, No.5 (2016)

Sussman T. and Bathe K.-J., A Finite Element Formulation For Nonlinear Incompressible Elastic and Inelastic Analysis, Computers and Structures, Vol. 26, No. 1/2 (1987), pp. 357-409. 